\title{
La vegetación como indicador de la Geomorfología Fluvial ${ }^{1}$
}

\author{
Silvina Lorena $\mathrm{Casco}^{2}$, Nora Indiana Basterra de Chiozzi ${ }^{3}$ y Juan José Neiff ${ }^{4}$ \\ ${ }^{1}$ Contribución del Proyecto PIP 815/PICT 12755 \\ ${ }^{2} 1$ Investigador Principal del Consejo Nacional de Investigaciones Científicas y Técnicas (CONICET) en el Centro de \\ Ecología Aplicada del Litoral (CECOAL). E-mail:_neiff@arnet.com.ar.Ruta 5 km 2,5. CC. 291. (3400) Corrientes. Argentina \\ Becaria Post-Doctoral del CONICET en el CECOAL. E-mail: sylvina@arnet.com.ar \\ ${ }^{3}$ Centro de Gestión Ambiental. Facultad de Ingeniería. Universidad Nacional del Nordeste E-mail: ibasterra@arnet.com.ar \\ ${ }^{4}$ Investigador Principal del CONICET en el CECOAL. E-mail: neiff@arnet.com.ar
}

\begin{abstract}
Resumo
Aguas abajo de la confluencia con el río Paraguay, el Paraná se expande en una ancha planicie lateral que, durante las inundaciones extraordinarias completa un área 2-10 veces mayor que el área ocupada por las aguas en los períodos de bajantes extraordinarias, en los que el curso queda aislado de la planicie inundable. El propósito de esta contribución es presentar las características más salientes de este tramo entrelazado del río, usando las unidades de paisaje y los tipos de vegetación, como indicadores de las formas del relieve. Imágenes satelitales Landsat TM5 y TM7 procesadas con el SIG IDRISI, de la inundación extrema de 1998 y de la sequía extrema de 2001, fueron usadas para la clasificación de los tipos de vegetación, usando el criterio propuesto por CET (Clasificación Ecológica de Tierras). Observaciones de campo fueron realizadas en ambas fases hidrológicas para obtener la posición topográfica de las unidades de vegetación y de los ensambles de comunidades vegetales. Nosotros encontramos que los albardones están ocupados por tipos de bosques, mientras que los bañados y cuerpos de aguas someros están cubiertos por plantas herbáceas y que las praderas flotantes de vegetación flotante libre, como el aguapé, crecen en lagos conectados al río una o más veces en el año. La vegetación acuática del curso principal es muy pobre como consecuencia del flujo del río y las fluctuaciones de nivel del agua. Sólo pequeñas manchas de carrizo se encontraron creciendo en barras de arena. La interrelación entre la vegetación y las formas del relieve es muy dinámica, con cambios drásticos en períodos de décadas o aún de pocos años, dependiendo del caudal y de la carga de sólidos transportados. En otro sentido, la vegetación fluvial tiene un importante rol en la fijación de depósitos fluviales y como núcleos de acreción en las barras o para entender la obstrucción de pequeños cursos de agua en la planicie de inundación. El estudio de la interrelación entre la vegetación y las formas del relieve proporciona un conocimiento sinóptico de la dinámica del paisaje, como indicador del régimen hidrológico en cada sitio y de los movimientos horizontales del agua que, finalmente son el principal factor que controla la erosión, transporte, acreción y la distribución y abundancia de los organismos.
\end{abstract}

Palabras chaves: Bajo Rio Paraná, vegetación fluvial, geoformas

\begin{abstract}
The Paraná River, downstream of the confluence with the Paraguay River, expands in to wide fringe floodplain that, during the extraordinary floods fill an area 2-10 times higher than the area occupied by waters in the extraordinary droughts, when the course is isolated from the floodplain. The purpose of this paper is to present characteristic features of the Paraná River braided system using the landscape units and the vegetation types as indicators of the land forms. Satelital images Landsat TM5 and TM7 from extreme flood of 1998 and extreme drought on 2001 were processed with a GIS IDRISI, to classify of vegetation types, according the ELC (Ecological Land Classification) criterias. Field observations were performed in both hydrological phases in
\end{abstract}


order to obtain the topographic position of the vegetation units and the assemblage of plant communities. We found that the levees are occupied by forest types while the wetlands and shallow lakes are covered by herbaceous plants, and the water hyacinth floating meadows are dominants in floodplain lakes connected with the river course one or more times in the year. The aquatic vegetation of the main course is very poor as a consecuence of the river flow and the water level fluctuations. Only small patches of reed stems were growing on sand bars. The relationship between vegetation and land forms is very dynamic with a drastic changes in periods of decades or still of few years, depending of the runoff and the amount of the suspended load. At the same time, the vegetation of this river has a very important rol in the fixation of river deposits and as accretion nuclei in the bars, or to understand the obturation of small streams in the flood plain. The study of the relationship between the vegetation and the relief allows a synoptic knowledge of the dynamics of the landscape, as indicator of the hydrological regime in each place of the course and of the horizontal movements of the water that finally, they are the main factor that controls the erosion, transport, accretion processes and the distribution and abundance of the organisms.

Key words: lower Parana River, fluvial vegetation, landforms

\section{Introducción}

Con frecuencia, el análisis geomorfológico de una cuenca o parte de ella, comienza con escalas pequeñas (1:500.000 a 1:100.000) dado que el análisisa nivel del paisaje, porque permite establecer la posible existencia de patrones de escurrimiento y la correspondencia con de diferentes los modelos de diseño fluvial, con características tales como declividad, suelos, vegetación. Estudios complementarios en escalas mayores permiten validar la posible relación entre escurrimientola red de avenamiento, las geoformas y la vegetación., los cuales constituyen la base para e ste diagnóstico del paisaje es la base para estudiar la variabilidad espacial y temporal de los ríos.

La utilidad de cada uno de estos tres elementos subsistemas, tiene valor como indicador diagnóstico sobrede los los demás, atributos del paisaje debido a que ambos interactúan en distinto grado (Fig. 1).

En tanto que en los paisajes de tierra firme, “clima y suelos, condicionan vegetación... yéstos a su vez, condicionan a la fauna ..." en las planicies fluviales, los cambios en la geomorfología y en la cantidad y calidad de agua, determinan las características de la vegetación que, a su vez, es la que produce la oferta de hábitat para la fauna y el soporte para las actividades humanas.

Las unidades (o manchas) del paisaje, se destacan en las imágenes a partir de diferencias espaciales y temporales en estos atributos.correlación entre las variables usadas como descriptores de los elementos del paisaje y que permiten establecer unidades de vegetación y paisaje. Hay un ajuste dinámico, regulado por las fluctuaciones energéticas del sistema (fluctuaciones hidrométricas y de caudal líquido) y por la cantidad de materiales que el río moviliza, transporta o acumula en cada sección de escurrimiento, con algunos procesos de feed-back entre los subsistemas representados en la figura 1, dando como resultado un diseño fluvial característico, que guarda una proporción variable de áreas emergidas y sumergidas para cada estado hidrológico del sistema.

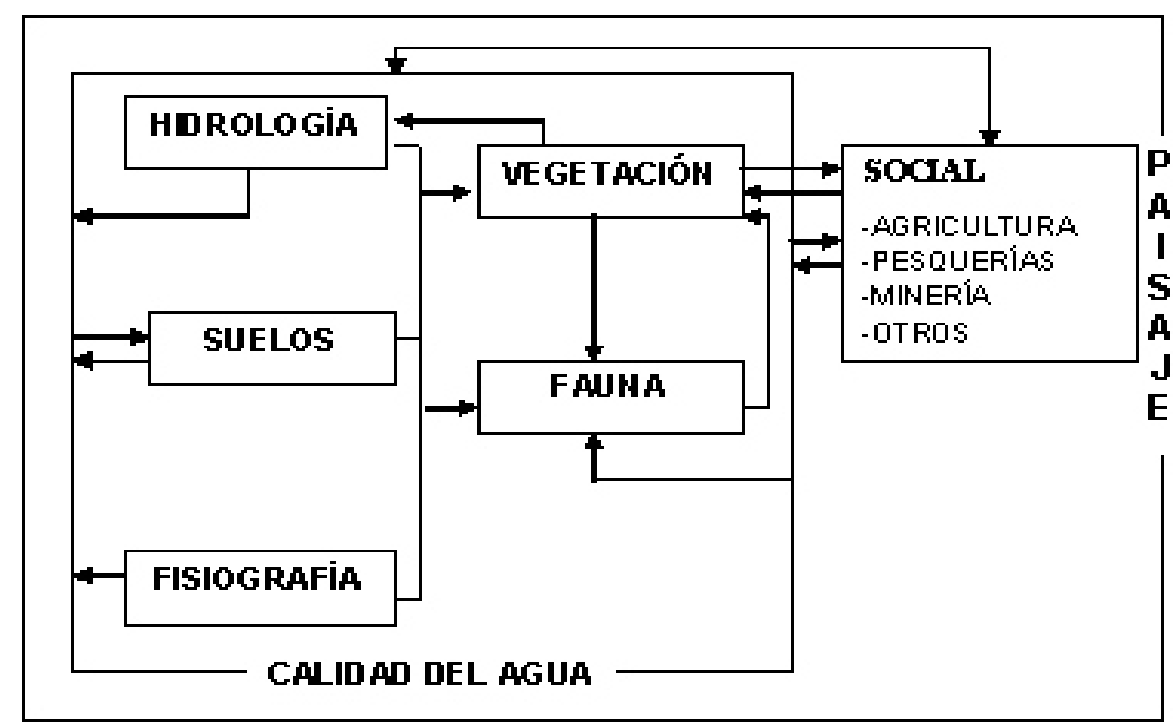

Fig. 1: Interacciones entre subsistemas del paisaje fluvial 
La influencia de la vegetación sobre las geoformas del paisaje fluvial es casi evidente. No todas las poblaciones dependen en igual grado de una u otra fase de los pulsos y, como consecuencia, hay patrones característicos de distribución desde el curso principal del río hasta las partes más altas de la varzea. A raíz de esto, existen diferentes combinaciones de árboles, arbustos y hierbas que se visualizan aún en las imágenes satelitales. En la práctica se pueden diferenciar y cuantificar estas unidades de paisaje (ecosecciones) y relacionarlas con el diseño geomorfológico del terreno, debido a que las plantas viven en una gama definida de condiciones de suelo inundado/suelo sumergido.

El efecto controlador de la erosión del canal por la vegetación ha sido reconocido y estudiado desde tiempo atrás (Wolman y Gerson, 1978; Newson, 1980). La estructura de la vegetación, especialmente la cobertura, densidad y estratificación de la vegetación riparia, es de crucial importancia en la magnitud y frecuencia de las inundaciones dado que producen cambios en el diseño del canal del escurrimiento (Petts, 1982). La existencia de vegetación firmemente establecida en los bordes del canal marca la magnitud de eventos de inundación que serían necesarios para producir cambios en la forma del canal (Graf, 1979).

Por otra parte, el establecimiento de vegetación permanente a las orillas del canal depende de factores hidrológicos (especialmente la variabilidad hidrométrica y la disponibilidad de humedad en el suelo) y geomorfológicos (como el grado de consolidación del suelo colonizable por plantas y la importancia de los procesos de erosión/sedimentación en cada punto de la planicie inundable).

$\mathrm{C}$ a mbios e n la di ná m i c a hidrosedimentológica del río podrían afectar el patrón de distribución de la vegetación y éste, a su vez, influir en la estabilidad de los taludes y en el efecto de "trampa" de sedimentos por parte de la vegetación. Modificaciones en el flujo, como ocurren aguas abajo de los embalses, la disminución de la velocidad del escurrimiento y la reducción del nivel de inundación determinó que los árboles (Salix spp. y Populus spp.) colonizaran hacia el centro del canal a lo largo del río Republican (Nebrasca; USA) lo que tuvo un importante efecto en la retención de una capa de sedimentos arenosos de hasta $1 \mathrm{~m}$ de espesor (Northrop, 1965). El área ocupada por sauces llegó a ocupar el $60 \%$ del canal lo que aumentó la rugosidad del suelo estimada a través de la fórmula de Manning de 0,036 (terreno arenoso sin vegetación) a 0,20 (suelo cubierto por árboles).

Dolan et al. (1974) registraron la invasión de Tamarix sp. y de Salix spp. en el río Colorado (USA) luego que el lavado de sedimentos desde los tributarios produjese un extenso abanico fluvial. Turner y Karpiscak (1980) informan de la estabilización y consolidación de bancos en el río Colorado, como consecuencia de la colonización de la vegetación sobre los abanicos fluviales. H oward y
Dolan (1981) encontraron que la deposición de sedimentos finos como arcillas y limos en el valle inundable del río Colorado están fuertemente relacionados con la densidad y cobertura de la vegetación. King (1961) señala que la vegetación prístina tiene efecto sobre la deposición y un efecto creciente en la consolidación durante futuras inundaciones. Ambos efectos cobran mayor importancia en los deltas fluviales (Kellerhals y Gill, 1973; Bergman y Sullivan, 1963).

Hearne y Armitage (1993) han cuantificado la influencia de la vegetación acuática en canales de escurrimiento mediante la aplicación de distintas ecuaciones. El incremento de biomasa de plantas produce una disminución de la sección libre del canal y pérdida de la capacidad de conducción, provocando desbordes laterales. Sin embargo, la erradicación de las plantas para evitar estos efectos podría producir aceleración del escurrimiento, aumento de la turbidez y cambios en la oferta de hábitat en los ríos estudiados por estos autores.

La vegetación de las varzeas de los grandes ríos tiene un importante efecto en la amortiguación del escurrimiento fluvial que hasta hoy es poco conocido. Neiff y Patiño (en prep.) estudiaron durante varios años la interferencia que la vegetación del Bajo Paraguay produce sobre las ondas de inundación y encontraron que la vegetación puede producir una disipación del volumen de agua pasado durante una inundación entre el 5 y el $20 \%$. Los valores más bajos fueron registrados cuando la frecuencia y duración de las inundaciones eran mayores e impedía el crecimiento del sotobosque y de la vegetación palustre. Cuando una inundación (de la misma magnitud) ocurría luego de un período largo de aguas bajas, se alcanzaban valores de disipación del escurrimiento próximos al $20 \%$, dado que la vegetación herbácea de la planicie determinaba mayores tiempos de residencia del agua. Neiff et al. (1994) demostraron el efecto que ejercen los bosques monoespecíficos densos de Tessaria integrifolia sobre el escurrimiento. En transecciones normales al eje de escurrimiento encontraron una disminución de velocidad de la corriente de 10 veces dentro del bosque, respecto de lugares aledaños no vegetados, en el curso del río. Este efecto, también tiene influencia sobre la erodabilidad de los bancos y sobre los procesos de acreción.

La vegetación de la planicie de inundación y del curso de ríos y arroyos tiene una importante función en la retención de sedimentos transportados por el río. En la vegetación flotante libre que crece en islas situadas en el curso del río las raíces de Eichhornia crassipes funcionan como un "peine" o filtro, reteniendo hasta $1326 \mathrm{~g}$ de sedimentos inorgánicos y hasta $2642 \mathrm{~g}$ por metro cuadrado (Poi de Neiff et al., 1994). Estos sedimentos son una fuente de nutrientes 
(Especialmente fósforo adherido a las partículas inorgánicas, que pasa rápidamente a ortofosfatos) lo cual permite el mejor crecimiento de las plantas. En este trabajo se explica que la cantidad de fósforo retenido por las plantas (adherido a los sedimentos) representa entre el 12 y el $31 \%$ del fósforo que necesitan las plantas durante su ciclo anual.

Una caída brusca de la velocidad del flujo debido a obras civiles (obras de navegación, represas) puede originar aumento de las tasas de sedimentación. Petrere y de Brito Ribeiro (1994) informan que la sedimentación luego de la represa de Tucuruí (cuenca Amazónica) ocasionó que 80-90\% de los sedimentos transportados por el río Tocantins quedaran retenidos en la represa. La retención de los nutrientes adheridos a los sedimentos en el tramo abajo de la represa determinó problemas de fertilidad en la vegetación que crece en las islas. Los cultivos de cacao y de palma debieron ser fertilizados como consecuencia de este impacto.

Esta contribución plantea analiza las posibilidades y limitaciones del estudiola utilización de la vegetación fluvial para definir estructuras de paisaje, especialmente las geoformas de la planicie del Paraná, aguas abajo de su confluencia con el Paraguay. Se analiza presenta la relación entre las formas del relieve (laposición topográfica de las barras) y lapresencia de distintos tipos de vegetaciónvegetación (unidades de paisaje, tipos de vegetación, bioformas dominantes), su distribución y abundancia.

\section{Métodos}

\section{1 Área de estudio}

La confluencia de los ríos Paraguay y Paraná se caracteriza por tener la estación estival calurosa y seca, con algunos días lluviosos hasta comienzos del otoño. En invierno las lluvias disminuyen y la temperatura desciende, aunque con baja ocurrencia de heladas (Bruniard, 1996, $1999 \mathrm{a}, \mathrm{b})$.

Geológicamente, integra la región denominada "Llanura Chaco-Pampeana", que constituye una unidad morfológica sin relieve, con poca movilidad de la corteza terrestre que se extiende por toda Sudamérica (Orfeo, 1995).

La zona de estudio comprendió $50 \mathrm{~km}$ en el sentido del curso del río Paraná, aguas abajo de la Confluencia con el río Paraguay, hasta la localidad de Empedrado, incluyendo las islas del curso (vinculadas a ambas márgenes) y a la planicie de inundación lateral, desde los $27^{\circ} 18^{\prime} 32^{\prime \prime}$ de latitud sur y $58^{\circ} 38^{\prime} 37^{\prime \prime}$ de longitud oeste hasta los $27^{\circ} 56^{\prime} 45^{\prime \prime}$ de latitud sur y $58^{\circ} 49^{\prime} 21^{\prime \prime}$ de longitud oeste (Fig. 2).

Utilizando imágenes Landsat $\mathrm{Tm} 7$, en

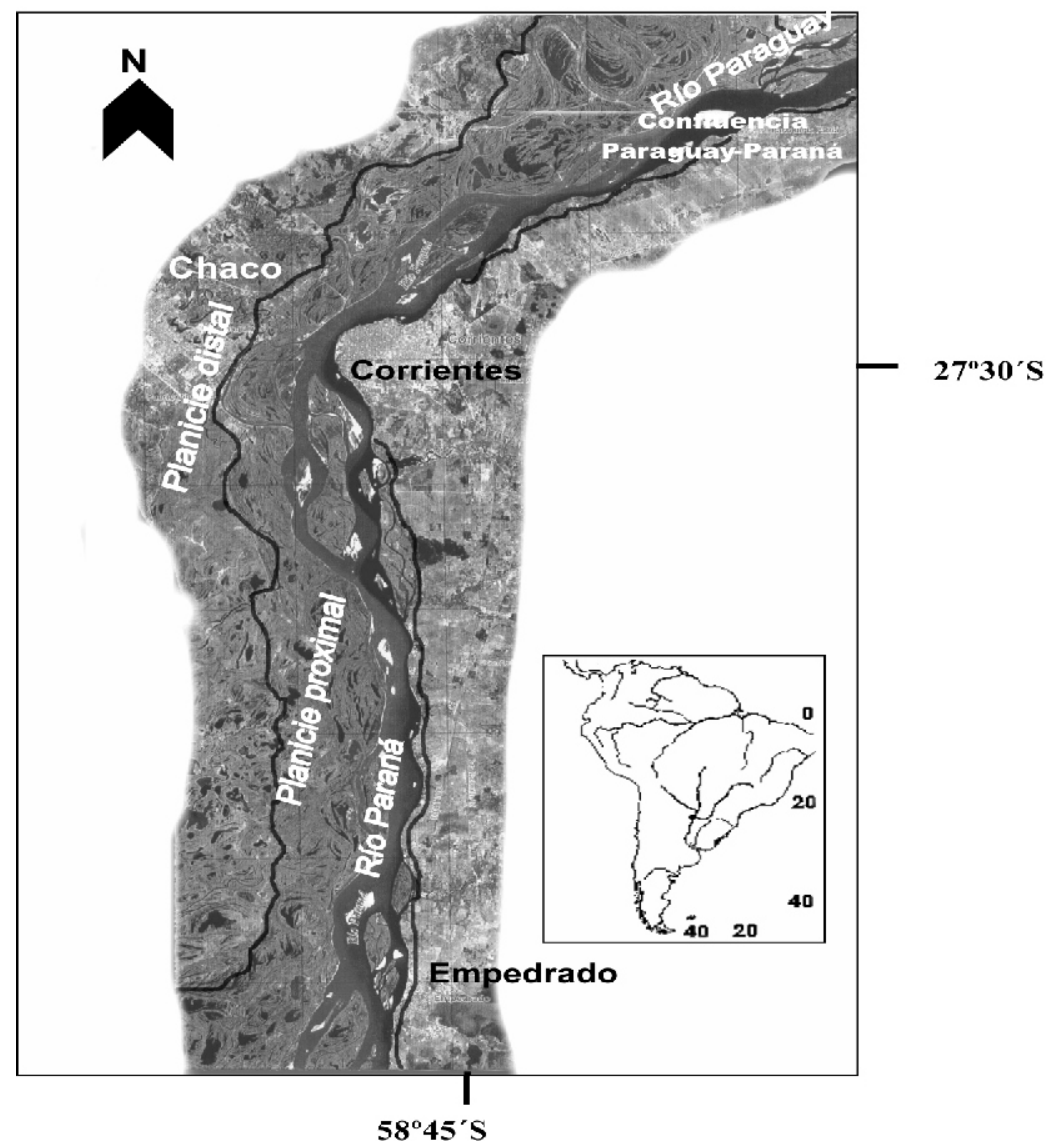

Figure 2: Ubicación del área de estudio. 
escala 1:250.000 se reconocieron las principales unidades geomorfológicas: curso de agua mayor, en cuyo recorrido se observan barras generalmente elongadas, de distinta altura y la planicie de inundación lateral, que es asimétrica, con mayor desarrollo sobre la margen derecha del río e incluye lagunas formadas por derrames laterales del Paraná y cuerpos de agua semilunares por derrame lateral de cursos secundarios (Orfeo, 1995, 1996; TGCC, 1996).

La planicie fluvial en este tramo del Paraná es suavemente ondulada, gradualmente más plana hacia el sur. Existe una diferencia máxima absoluta de 7,66 metros en Corrientes y sólo de 3,20 metros en Empedrado, al comparar la parte más baja del relieve y la cúspide de las islas más altas.

La planicie aluvial puede ser diferenciada por el tono y textura en las imágenes satelitales, en dos grandes subunidades: planicie de inundación proximal, franja adyacente al curso, situada hacia la margen derecha e influenciada por las crecientes de menor intensidad (anuales) y planicie de inundación distal, situada sobre la margen derecha en este tramo, aislada del curso del río por la planicie proximal y afectada sólo por crecientes extraordinarias (Orfeo, 1995).

El paisaje de la zona estudiada ha sido y es constantemente modificado y remodelado por el río, especialmente en las islas del curso, que modifican el tamaño, forma y posición de las barras, en períodos menores que una década, como consecuencia de las grandes crecientes.

La vegetación del área está formada por plantas arbóreas, arbustivas y herbáceas en distinta agregación, de acuerdo a la amplitud del relieve y a la disponibilidad de agua en y sobre el suelo (Neiff, 1986; Casco, 2003).

\subsection{Reconocimiento en escala satelital}

Se analizaron imágenes en formato digital, proporcionadas por CONAE (Comisión Nacional de Actividades Espaciales- Argentina, entre las ciudades de Corrientes y Empedrado (Fig. 2) separadas por $68 \mathrm{~km}$. Se compararon dos fases hidrológicas contrastadas: aguas altas extraordinarias, influenciada por el fenómeno "El Niño", (imagen del 4 de mayo de 1998) y para la época de aguas bajas extraordinarias coincidente con el fenómeno "La Niña" (24 de agosto de 2001) tomadas por el sensor Thematic Mapper (TM) del satélite Landsat 5 (aguas altas) y Landsat 7 (aguas bajas) y path 226, row 079 del Digital Worldwide Reference System.

Las imágenes posuen una resolución espacial de $30 \mathrm{~m}$ y una resolución espectral de 7 bandas del espectro electromagnético. Seis bandas pertenecen al espectro visible e infrarrojo reflejado (azul, verde, rojo, e infrarrojo cercano y medio) y u n a l infrarrojo térm i co. Las imágenes fueron procesadas con el software IDRISI (Universidad de Clark, USA), tomándose como base para la identificación de las unidades de paisaje la correspondiente a aguas bajas (agosto del 2001), debido a que en esta condición fue posible encontrar el espectro más amplio de parches distintos por su tonalidad, forma y extensión.

En la imagem de fato color compuesta, se identificaron visualmente y se digitalizaron las unidades de paisaje más representativas. En cada parche homogéneo se extrajeron las firmas espectrales que luego fueron utilizadas por el software en la clasificación.

En ambas imágenes (potamofase y limnofase), se calculó el porcentaje ocupado por agua en la zona seleccionada durante ambas fases hidrológicas.

\subsection{Reconocimientos en campo}

El análisis de la vegetación fluvial se realizó en dos oportunidades: 1998) y

- en aguas altas (septiembre-noviembre de

- en aguas bajas (julio-noviembre de 1999)

En ambas oportunidades, se reconocieron las islas del curso y la planicie de inundación del Bajo Paraná.

Para la clasificación de las unidades de paisaje se utilizó el sistema de Clasificación Ecológica de Tierras (ELC: Ecological Land Classification) empleado por FEARO (1978), que consiste en considerar las relaciones temporales y espaciales entre los cinco componentes principales del paisaje: terreno, hidrología, clima, suelo y vegetación, permitiendo identificar las superficies terrestres que posean semejanzas en su paisaje y determinar zonas geográficas con diferente nivel de detalle que conforman el patrón de paisaje (TGCC, 1996; Basterra, 1999; Casco, 2003).

Luego de la clasificación preliminar de las unidades de paisaje en las imágenes, se estudió la distribución de la vegetación fluvial en cada una de ellas, tomando la posición topográfica con respecto al nivel de la lámina de agua, asociándola con los datos del puerto de Corrientes y de Empedrado en el momento de la medición y corrigiéndola luego respecto del nivel del mar (Neiff, 1986).

En el momento en que el río se encontraba en su máximo nivel, se realizó un amplio reconocimiento de las islas, con un bote de cinco metros de eslora, con motor y una plomada dotada de una cuerda fina graduada a intervalos de $20 \mathrm{~cm}$. En cada punto a "nivelar" en las islas, se tomaba la profundidad desde el suelo de la isla al "pelo del agua" en ese momento.

En gabinete, las medidas de campo fueron referidas al cero hidrométrico de la cota de los puertos Corrientes y Empedrado, proporcionado por la Dirección Nacional de Construcciones Portuarias y Vías Navegables, para las islas 
próximas a los mismos.

Para ilustrar el procedimiento, un punto de la isla Chouí que se encontrara a $-3,00 \mathrm{~m}$ de profundidad respecto del nivel actual del "pelo de agua" en Corrientes, le correspondería una cota de 44,39 m respecto del nivel del mar (cero del Riachuelo) según el siguiente procedimiento:

HIDRóMETRO CORRIENTES, NIVEL ACTUAL: $5,00 \mathrm{~m}$

MEDIDA DE CAMPO en determinado punto de registro: 3,00 $\mathrm{m}$ de profundidad desde la lámina de agua $(=-3,00)$.

NIVEL DEL HIDRóMETRO DE CORRIENTES (respecto del nivel del mar): $42,39 \mathrm{~m}$

$42,39+(5,00 \mathrm{~m}-3,00 \mathrm{~m})=44,39$ m.s.n.m.

Este trabajo de campo, ayudado con un georreferenciador (GPS), permitió colocar las cotas de terreno en la imagen y establecer la posición topográfica en la cual se encontraban las distintas unidades de paisaje identificadas.

Se logró un registro con información del tipo de paisaje presente, las poblaciones vegetales que allí se encontraban y su posición topográfica. Esto permitió obtener la frecuencia de cada población (especie) en cada sitio del gradiente topográfico y elaborar las curvas que sintetizan la distribución de la vegetación según la topografía del terreno.

Para tener una valoración sinóptica de la variabilidad del sistema, se calculó el Cociente de Elasticidad (Neiff et al., 1994) en tres secciones del área de estudio, midiendo el ancho del área mojada por las aguas del río en las imágenes satelitales. La razón entre potamofase y limnofase fue encontrada mediante IDRISI.

La cantidad de días con suelos inundado o seco y su influencia en la distribución de la vegetación y en el modelado de las geoformas, fue estimada a través del Cociente de Conectividad Fluvial FCQ- (Neiff y Poi de Neiff, 2002), como:

\section{$F C Q=\underline{N^{0} \text { de días en inundación }}$ $N^{0}$ de días en aislamiento}

Se analizó la serie hidrométrica 1997-2002 con el software Pulso (Neiff y Neiff, 2003), evaluando la frecuencia, intensidad, tensión, recurrencia, amplitud y estacionalidad del pulso hidrosedimentológico (Neiff, 1990), indicadores de la función Fitras, que influyen localmente en la vegetación. Se tomaron los niveles de desbordes más frecuentes para cada unidad de paisaje.

\section{Resultados}

El análisis de las imágenes satelitales y los reconocimientos de campo, permitieron diferenciar unidades de paisaje con poblaciones vegetales arbóreas y herbáceas, situadas en geoformas distintas (Fig. 3).
Las unidades de paisaje con vegetación herbácea $\left(\mathrm{A}_{2}, \mathrm{~B}_{2}, \mathrm{~B}_{3}\right.$ y $\left.\mathrm{C}\right)$ y leñosas $\left(\mathrm{F}_{1}, \mathrm{~F}_{2}\right)$ se encontraron en todo el gradiente topográfico de las islas sin embargo, las herbáceas fueron más frecuentes y con mayor cobertura en posiciones topográficas más bajas, mientras que los paisajes con poblaciones leñosas ocuparon sitios más altos, menos variables en el tiempo (Fig. 4).

En la planicie de inundación se encontraron numerosas lagunas $\left(\mathrm{A}_{2}\right)$ de forma semilunar (madrejones), formadas por unión de barras de arena, donde predominó la vegetación flotante libre que puede ocupar $80 \%$ de la superficie del agua. Entre 1997 y 2002 permanecieron inundadas 579 días, con frecuencia de 20 pulsos y un FCQ de 0,37 . Las lagunas ubicadas en las islas, recibieron una frecuencia de 30 pulsos (918 días en aguas altas) y el cociente de conectividad fluvial fue de 0,78 (Tabla 1).

Los bañados con plantas altas y tiernasgraminoides y latifoliadas- $\left(\mathrm{B}_{2}\right)$ ocuparon áreas cóncavas que se forman en el interior o en la cola de las islas, como consecuencia del elevamiento de los albardones o por el adosamiento de los bancos de arena. Los suelos estuvieron 560 días bajo agua, con un valor más bajo de FCQ que las lagunas isleñas $(0,35)$.

Los bañados con pastos altos y durospajonales y cañaverales- $\left(\mathrm{B}_{3}\right)$ se encontraron en suelos más altos que los de $\mathrm{B}_{2}$, por lo cual estuvieron menor número de días inundados (450). Ocuparon microdepresiones centrales de las islas y extensos sectores de la planicie de inundación, conectándose con el río menos frecuentemente que las unidades de paisaje anteriormente mencionadas $(12, \mathrm{FCQ}=0,26)$.

El mayor número de especies (52) se registró en posiciones bajas del gradiente topográfico (46,8 m.s.n.m.), de las cuales fueron más frecuentes aquellas características de bañados $\left(\mathrm{B}_{1}, \mathrm{~B}_{2} \mathrm{y} \mathrm{B}_{3}\right)$ debido a su rápido crecimiento $\mathrm{y}$ alta productividad.

Los bañados con arbustos y con palmas $\left(\mathrm{B}_{4}, \mathrm{~B}_{5} / \mathrm{B}_{6}\right)$ se situaron en la planicie de inundación (levemente cóncava) y estuvieron bajo agua sólo 356

días (Tabla 1), de los cuales el 50\% correspondió al año 1998 (creciente extraordinaria, efecto de "El Niño"). Su FCQ fue uno de los menores hallados (0,22). Los palmares de Copernicia alba se distribuyeron en un rango más restringido del gradiente topográfico, comprendido entre 45,3 y 49,3 m.s.n.m. (Fig. 5). Los esteros y juncales (C) también ocuparon bañados bajos situados en la planicie, permaneciendo la mayor parte del tiempo con el suelo seco (1581 días, Tabla 1). 


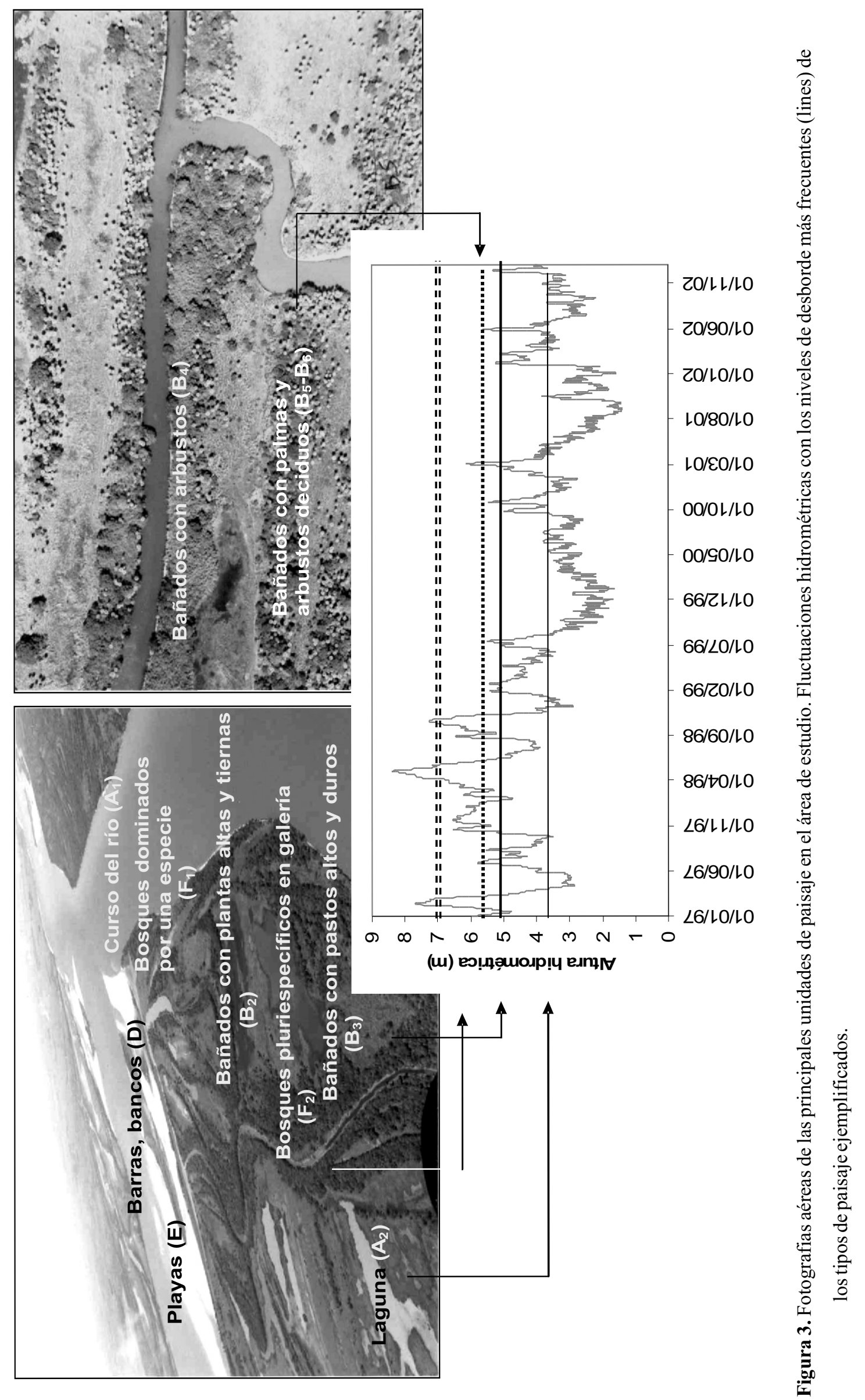




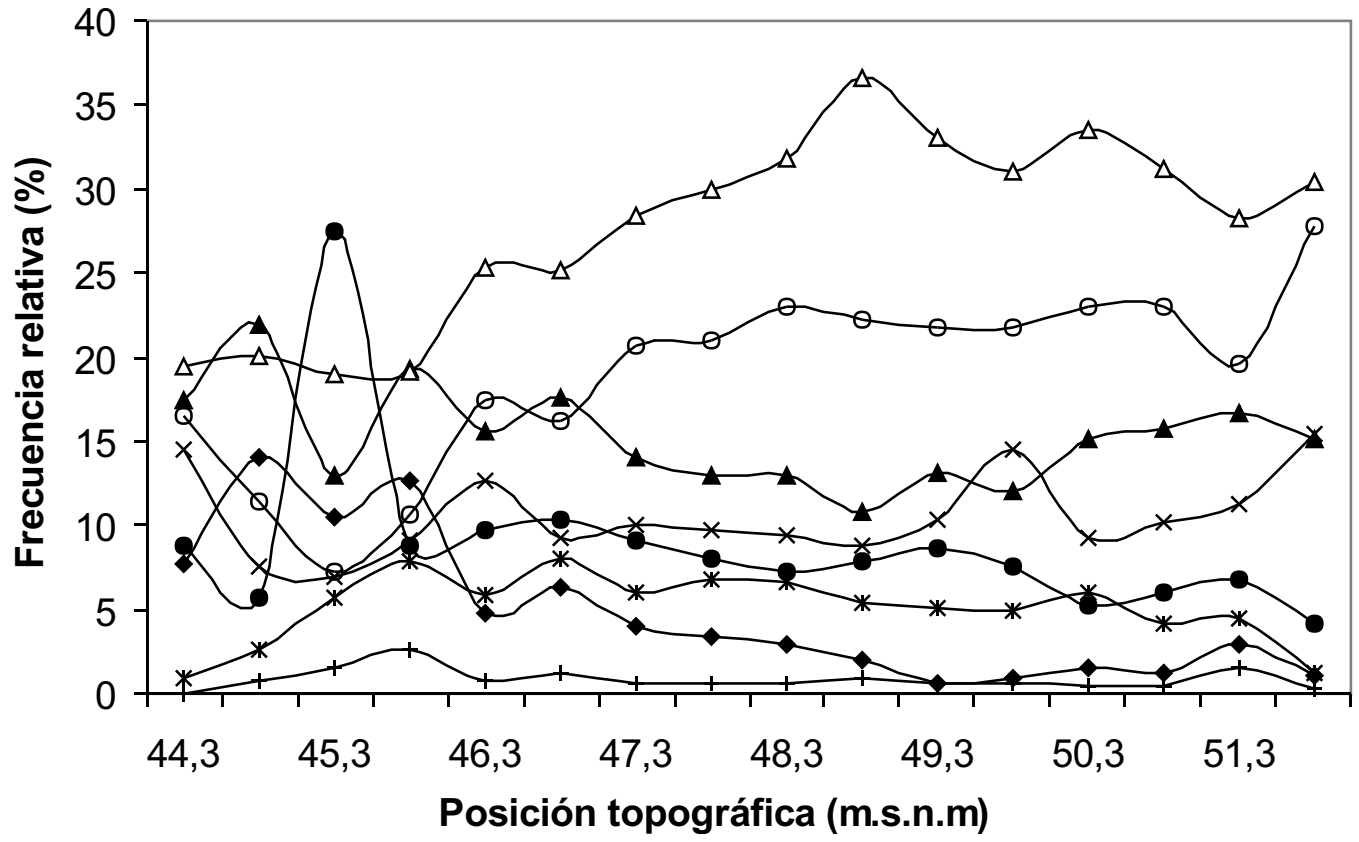

$\rightarrow \mathrm{A} 2 \multimap \mathrm{B} 2 \rightarrow \mathrm{B} 3 \rightarrow \mathrm{B} 4 \rightarrow \mathrm{B} 5 / \mathrm{B} 6 \rightarrow \mathrm{C} \rightarrow \mathrm{F} 1 \rightarrow \mathrm{F} 2$

Figure. 4: Distribución de las principales unidades de paisaje del área de estudio en el gradiente topográfico

Tabla 1: Atributos del pulso hidrosedimentológico en las principales unidades de paisaje del Bajo Paraná durante el período 1997-2002

\begin{tabular}{|c|c|c|c|c|c|c|c|c|c|}
\hline & $\begin{array}{c}\mathbf{A}_{2} \\
\text { (planicie) }\end{array}$ & $\begin{array}{c}\mathbf{A}_{2} \\
\text { (isla) }\end{array}$ & $\mathbf{B}_{1}$ & $\mathbf{B}_{2}$ & $\mathbf{B}_{3}$ & $\mathbf{B}_{4} / \mathbf{B}_{5} / \mathbf{B}_{6}$ & $\mathrm{C}$ & $F_{1}$ & $\mathbf{F}_{2}$ \\
\hline Frecuencia & 30 & 20 & 18 & 20 & 15 & 12 & 15 & 8 & 3 \\
\hline Intensidad máxima & 8,39 & 8,39 & 8,39 & 8,39 & 8,39 & 8,39 & 8,39 & 8,39 & 8,39 \\
\hline Intensidad mínima & 1,4 & 1,4 & 1,4 & 1,4 & 1,4 & 1,4 & 1,4 & 1,64 & 2,85 \\
\hline Tension máxima & 6,40 & 6,22 & 6,40 & 6,22 & 6,22 & 6,22 & 6,22 & 5,92 & 1,89 \\
\hline Tension mínima & 11,01 & 11,01 & 11,01 & 11,01 & 11,01 & 11,01 & 11,01 & 10,58 & 4,83 \\
\hline Recurrencia & 10 & 4 & 8 & 11 & 6 & 11 & 6 & 3 & 3 \\
\hline Amplitud & 69,7 & 98,4 & 115,9 & 98,4 & 144 & 164,3 & 131,1 & 84,4 & 209 \\
\hline $\begin{array}{c}\text { Estacionalidad } \\
\text { Potamofase }\end{array}$ & feb-mar & feb & feb-mar & - & - & - & - & - & - \\
\hline $\begin{array}{l}\text { Estacionalidad } \\
\text { Limnofase }\end{array}$ & $\begin{array}{l}\text { ene, abr - } \\
\text { dic }\end{array}$ & $\begin{array}{l}\text { ene, } \\
\text { mar-dic }\end{array}$ & $\begin{array}{l}\text { ene, abr- } \\
\text { dic }\end{array}$ & $\begin{array}{l}\text { ene- } \\
\text { dic }\end{array}$ & $\begin{array}{l}\text { ene- } \\
\text { dic }\end{array}$ & ene-dic & ene-dic & $\begin{array}{l}\text { ene- } \\
\text { dic }\end{array}$ & $\begin{array}{l}\text { ene- } \\
\text { dic }\end{array}$ \\
\hline $\begin{array}{l}N^{0} \text { de dias en } \\
\text { potamofase }\end{array}$ & 918 & 579 & 680 & 560 & 450 & 356 & 388 & 271 & 87 \\
\hline $\begin{array}{l}N^{0} \text { de dias en } \\
\text { limnofase }\end{array}$ & 1175 & 1585 & 1420 & 1604 & 1714 & 1615 & 1581 & 1252 & 540 \\
\hline FQC & 0,78 & 0,37 & 0,48 & 0,35 & 0,26 & 0,22 & 0,25 & 0,22 & 0,16 \\
\hline
\end{tabular}




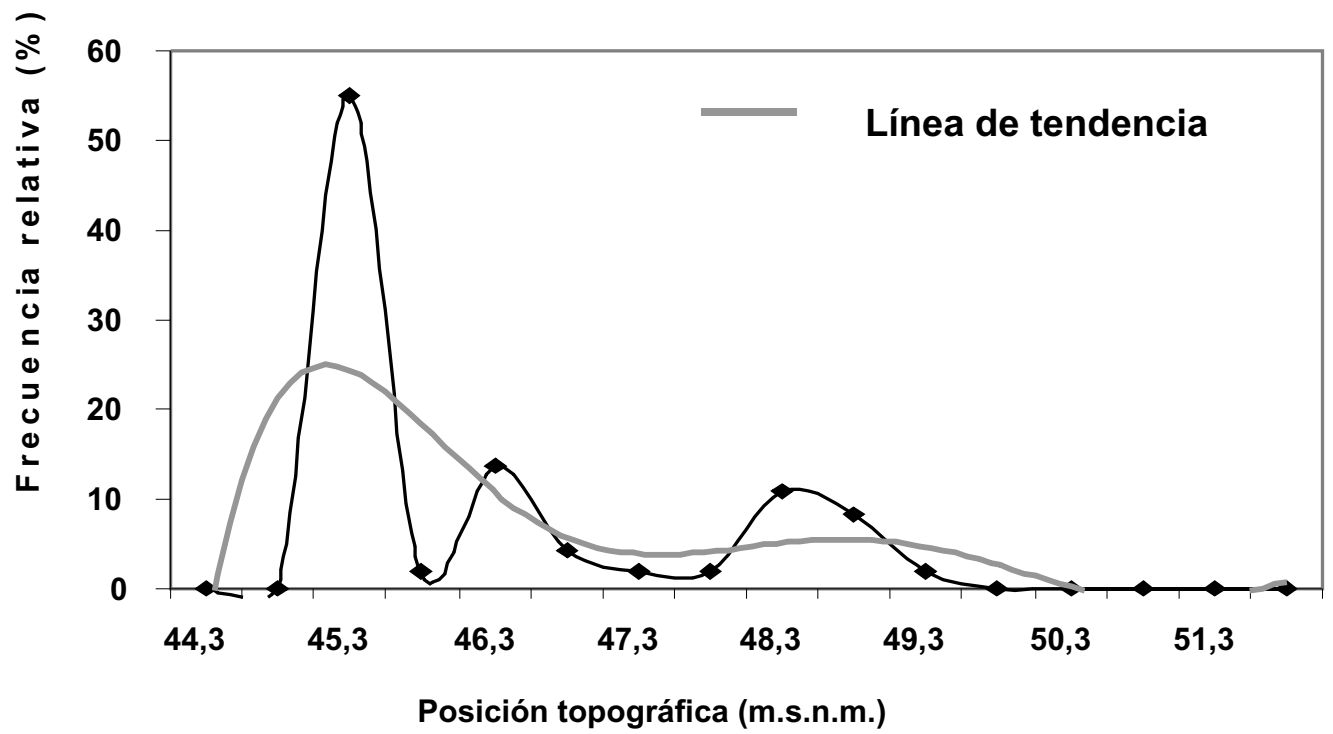

Figure 5: Distribución de los palmares de Copernicia alba en la planicie de inundación del Bajo Paraná

Los bosques dominados por Salix humboldtiana y Tessaria integrifolia $\left(\mathrm{F}_{1}\right)$ colonizaron los bancos de arena (D) y se situaron en islas ubicadas en diferentes posiciones topográficas. Los sauces fueron más frecuentes (19\%) en 47,3 m.s.n.m., mientras que los alisos ocuparon más frecuentemente $(22,08 \%)$ sitios un metro y medio más altos (Fig. 6). Estos bosques estuvieron conectados con el río a través de 8 pulsos, que

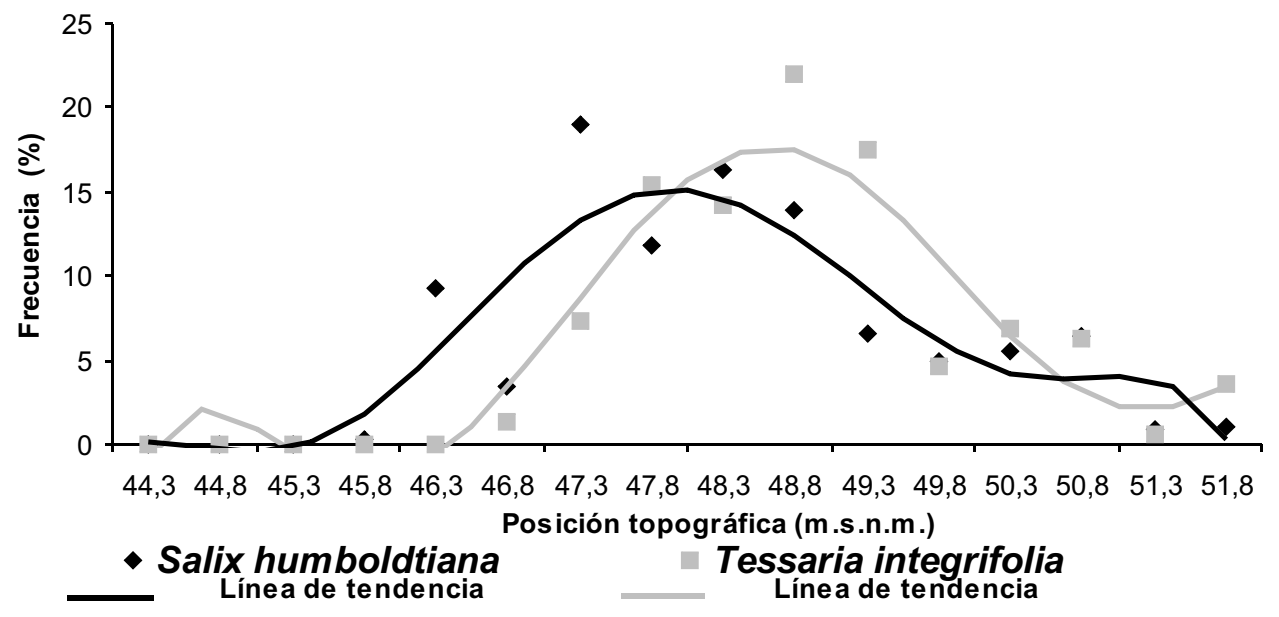

Figure. 6: Distribución de las poblaciones de sauces y alisos en el área de estudio 
incluyen 271 días bajo agua (Tabla 1).

Los bosques pluriespecíficos $\left(\mathrm{F}_{2}\right)$ se localizaron en albardones altos del curso principal e islas y en albardones de la planicie lateral de inundación. Fueron más frecuentes $(36,55 \%)$ en 48,8 m.s.n.m. (Fig. 3). Este tipo de bosque permaneció con el menor tiempo de suelo inundado (87 días) y, por lo tanto, registró el menor FCQ $(0,16)$.

El análisis con IDRISI de la imagen de aguas altas, en la zona cercana a Empedrado, proporcionó un valor de $75,7 \%$ de la superficie ocupada por el río. Sin embargo, en reconocimientos de campo pudo comprobarse que el río ocupó $95 \%$ de la zona (Fig. 7 a), quedando emergentes sólo las unidades de paisaje que se situaron en suelos más altos (lagunas permanentes, bañados con plantas altas y tiernas y bosques de sauces/alisos y pluriespecíficos, pastizales y áreas agropecuarias). Esta diferencia se debe a que sólo la superficie ocupada por los troncos de los árboles no estaba bajo agua, de tal manera que el programa los considera como tierra firme.

Tal como se observa en la imagen satelital en

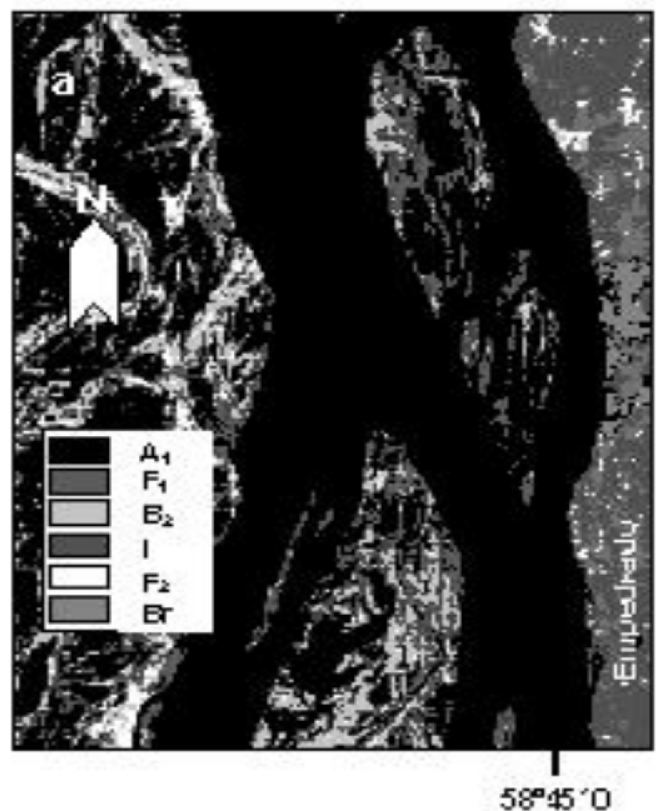

época de bajante (Fig. 7 b), la vegetación fluvial presenta un patrón de paisaje más complejo debido a la recolonización de las especies herbáceas, cuya distribución y abundancia se relaciona con la posición topográfica que ocupan. La vegetación flotante libre (Eichhornia spp.; Pistia stratiotes; Salvinia biloba) queda restringida a los cuerpos de agua permanente; las plantas herbáceas altas y tiernas $\left(\mathrm{B}_{2}\right)$ a los bañados más inundables y los pajonales de Panicum prionitis $\left(\mathrm{B}_{3}\right)$ recolonizan en áreas más altas, inundadas sólo por unos meses en el año. Las áreas agropecuarias (I) también se situaron en suelos más altos, siendo frecuentes los cultivos como microemprendimientos familiares.

El área de estudio puede ser considerada como de elasticidad alta, en cuenta que el ancho del área mojada, tiene mucha diferencia al comparar el estado de máxima inundación (Fig. 8 a), con las transecciones tomadas en la imagen satelital correspondiente a la máxima sequía (Fig. 8 b), encontrándose una razón más frecuente de 5:1 hasta de 11,5:1.

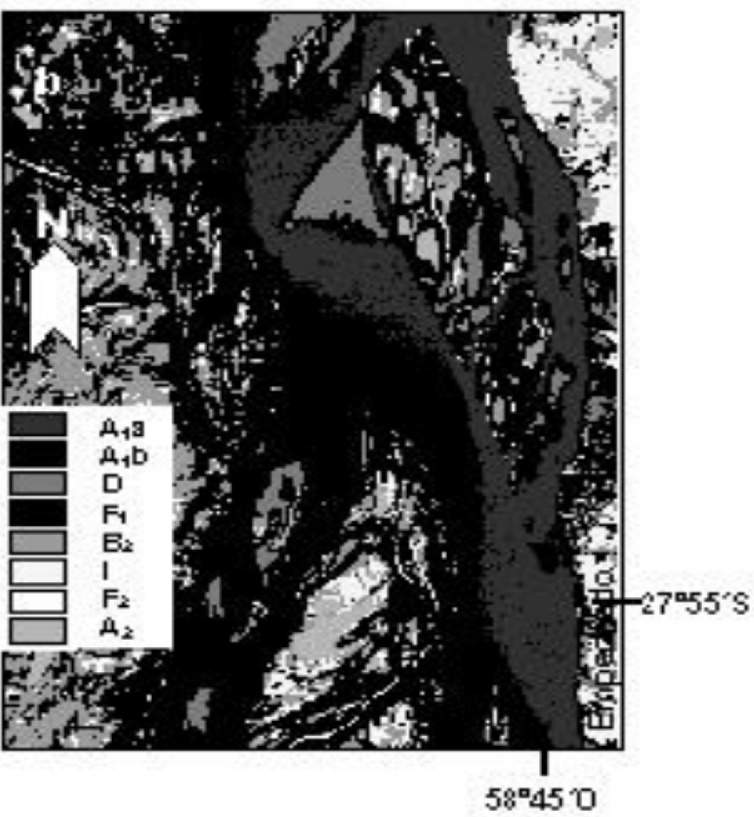

Figure 7: Imágenes satelitales de la sección del área de estudio cercana a la localidad de Empedrado mostrando el patrón de distribución de las unidades de paisaje en potamofase (a) y en limnofase (b). $\mathrm{A}_{1}$ : curso del río; $\mathrm{A}_{1}$ a: aguas claras; $\mathrm{A}_{1} \mathrm{~b}$ : aguas blancas; $\mathrm{A}_{2}$ : lagunas; $\mathrm{B}_{2}$ : bañados con plantas altas y tiernas; $\mathrm{Br}$ : barranca; $\mathrm{D}$ : barras de arena; $\mathrm{F}_{1}$ : bosques dominados por sauce o por aliso; $\mathrm{F}_{2}$ : bosques pluriespecíficos; I: pastizales y áreas agropecuarias.

\section{Discusión y Conclusiones}

La dinámica geomorfológica, la calidad de las aguas, la relación de áreas inundadas, inundables y emergidas y las características de los sedimentos do que proporcionan rasgos particulares al Bajo Paraná han sido reconocidas por otros autores (Neiff, 1990; 1999; Orfeo y Stevaux, 2002).

Espacialmente, el diseño de escurrimiento y la secuencia de flujo y reflujo de agua, sedimentos y organismos tienen diferencias locales que se reflejan en la 

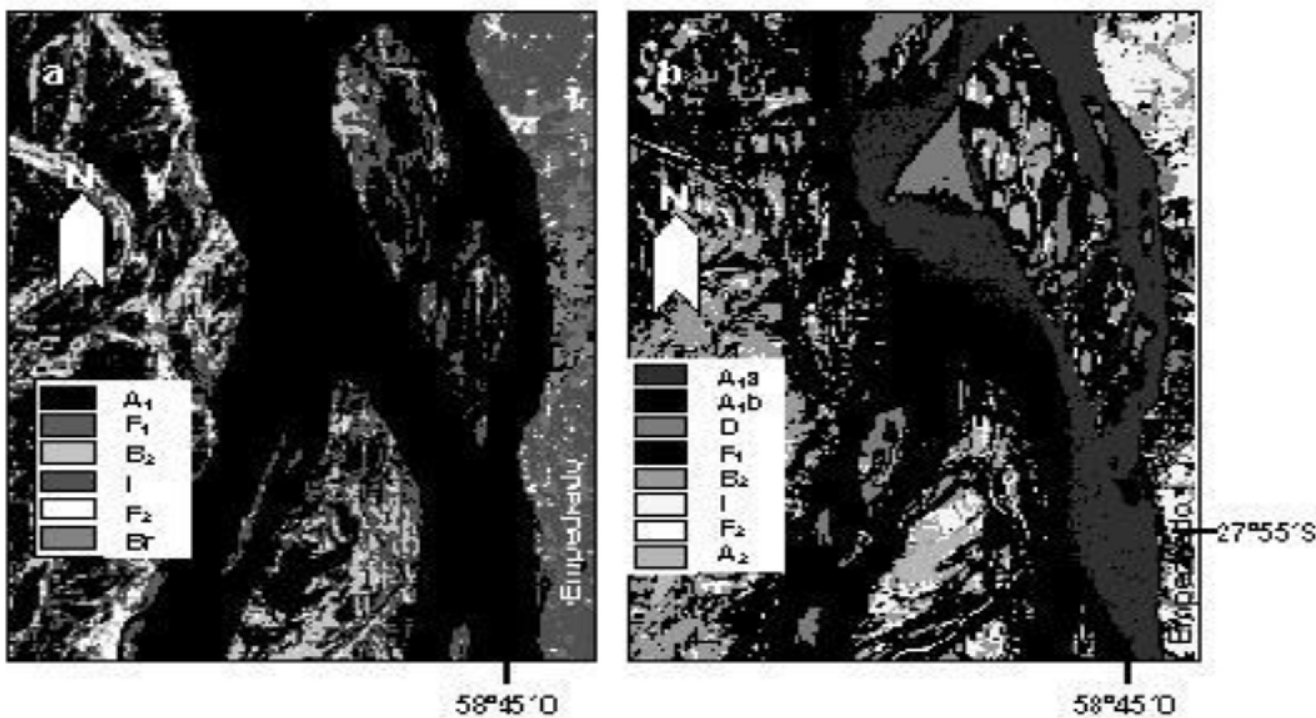

Figure 8: Imágenes satelitales del área de estudio con los cocientes de elasticidad correspondientes a cada transección (1, 2 y 3). a) Fase de aguas altas; b) Fase de aguas bajas.

diferencias locales que se reflejan en la distribución de las unidades de paisaje.

La posición, forma y superficie de cada unidad de paisaje indica una relación con la posición topográfica de cada sitio. De acuerdo a Bravard y Gilvear (1996) la relación entre el canal de un río y el conjunto de variables ambientales determina estilos geomorfológicos, con hábitat especiales y las discontinuidades están influenciadas por el funcionamiento ecológico del río y su planicie aluvial adyacente.

Hay un entorno de probabilidades de configuración del paisaje que está determinado por el banco de semillas remanentes en el suelo, por el aporte de semillas que trae el río y por otros factores de acción local como la formación de barras, en las que se depositan y concentran gran cantidad de semillas por efecto del viento y la dirección del escurrimiento entre otros factores, lo que luego, darán origen a bosques de aliso, de sauce u otro paisaje.

El análisis de imágenes satelitales correspondientes a situaciones hidrológicas muy contrastadas en un mismo ciclo, dan cuenta que la vegetación herbácea es más sensible a estos cambios de estado. Sin embargo, el análisis de series largas de tiempo, permite conocer que la vegetación tiene una alta resiliencia, que le permite mantener determinado patrón de organización en cada tramo del río.

No todas las poblaciones dependen en igual grado de una $\mathrm{u}$ otra fase $\mathrm{y}$, como consecuencia, se observan patrones pan distintas característicos de distribución desde el curso principal del río hasta las partes más altas de la varzea. De tal manera, en las imágenes satelitales es posible establecer unidades de mapa dominadas por determinado patrón de organización en el que árboles, arbustos y hierbas ocupan distintas proporciones. En la práctica se pueden diferenciar y cuantificar estas unidades de paisaje (ecosecciones), aún cuando los límites no siempre sean bien definibles, debido a posibles transiciones entre dos o más ecosecciones.

Cada una de estas ecosecciones tiene un conjunto de formas de vida o bioformas que la distinguen de otras unidades de paisaje. Sin embargo hay un grupo menor de plantas que pueden ocupar varias unidades de paisaje configurando estas transiciones.

La amplitud de fase también condiciona el espectro biológico de la vegetación. En tanto aumente la duración de la potamofase, tenderán a dominar las bioformas flotantes en las lagunas y bañados de la varzea del Paraná. Cuando la limnofase tiene una duración superior a la "normal" comienzan a dominar las bioformas arraigadas emergentes (Neiff, 1978). Moschini et al. (1995) encontraron que la potamofase favorece el crecimiento de las plantas de Scirpus sp. las cuales alcanzan ventajas en la colonización de los cuerpos de agua de la cuenca del Mogí Guaçu (afluente del alto Paraná.)

La mayor duración de la fase de inundación puede producir stress grave en las plantas como consecuencia de la falta de oxígeno en las raíces 
(hipoxia, anoxia), tal como señalan Whitlow y Harris (1979). Los síntomas de stress son muy variados: inhibición de la floración y de la fructificación, defoliación y, finalmente, muerte de las plantas.

Tal como expresara Whittaker (1978), la complejidad de la topografía y otros factores ambientales espaciales componen el patrón de la vegetación en el que puede esperarse solapamiento de las curvas de distribución, en tanto las poblaciones sean anfitolerantes. La presencia de determinado paisaje o población de la vegetación fluvial depende principalmente de las condiciones del medio físico para permitir o no la germinación. Luego de la ecesis (etapa de colonización del suelo), las posibilidades de la vegetación para resistir sequías e inundaciones extremas son altas, lo que determina la persistencia del patrón de paisaje aun cuando se produzcan condiciones hidrológicas muy contrastadas en un mismo sitio.

En ríos con regímenes altamente estacionales se encuentran condiciones de grandes crecientes y bajantes extremas dentro del lecho arenoso del canal, generando una selección de la vegetación y permitiendo sólo la permanencia de aquellas bioformas mejor adaptadas (Wende y Nanson, 1998).

Durante las crecientes extraordinarias, gran parte de las islas del Bajo Paraná quedan sumergidas, incorporándose a la sección de escurrimiento. Sin embargo, cuando el suelo queda descubierto de agua, los bancos son colonizados por plantas pioneras cuyo efecto protector es efectivo (Orfeo, 1995). Esta característica fue observada también en otros ríos, como el Durack (Australia) y el Narew (Polonia), donde la protección natural que ejerce la vegetación riparia controla la erosión de los canales (Wende y Nanson, 1998; Gradzi $\square$ ski et al., 2003).

Durante este estudio, ninguna de las especies vegetales que constituyen las unidades de paisaje fue encontrada germinando en condiciones de inmersión prolongada, en grandes ríos (Neiff, 1999; Junk y Piedade, 1993) esta evidencia señala que las plantas pueden colonizar un sitio de la planicie aluvial sólo en algunas posiciones del gradiente topográfico. También las sequías e inundaciones extremas, que se presentan en secuencias plurianuales (García y Vargas, 1998) indican que el área ocupada por paisajes dominados por plantas leñosas o herbáceas puede desplazarse temporalmente (Neiff, 1990; Casco, 2003).

Los resultados de este estudio indican que las unidades de paisaje con predominancia de especies leñosas son algo más frecuentes en los sitios más altos del área de estudio, en tanto que la vegetación herbácea se distribuye con mayor frecuencia en sitios más bajos del gradiente. Sin embargo, tanto leñosas como herbáceas, tienen curvas de distribución muy amplias en el gradiente topográfico, lo que permite explicar que el paisaje fluvial se mantiene como corredor discernible aún en imágenes satelitales, al atravesar ecosistemas terrestres tan distintos (Sabana Chaqueña, Espinal, Estepa pampeana).

En el área de este estudio la planicie de inundación tiene fluctuaciones hidrométricas muy importantes, como lo demuestran los valores del cociente de elasticidad. Esta característica, debida principalmente a la geomorfología plana y a las variaciones del nivel hidrométrico del río, ha determinado un proceso de selección natural que favoreció a aquellos organismos que pudieran absorber la amplitud de fluctuación del ambiente. Por lo tanto muchas especies de plantas y animales se comportan como transgresivas encontrándoselas en distintas unidades de paisaje, a pesar de encontrar su óptimo vital en determinada posición topográfica de la planicie.

Los cambios en la vegetación se producen en cortos períodos, de décadas o de años, por lo que el paisaje nunca alcanza su etapa "clímax" (Franceschi y Lewis, 1979) y la sucesión que se opera en las formas del relieve y en la vegetación que las cubre, no puedan ser interpretadas según una dirección definida como en la concepción de Clements (Neiff, 1990 y 2003).

En el Bajo Paraguay (Neiff et al.,en prensa) la vegetación produce una amortiguación marcada de las crecidas al retardar el escurrimiento y disipar grandes volúmenes de agua que no llegan al tramo bajo. La disminución de la velocidad del flujo produce una reducción de la tasa erosiva por el agua. El efecto atenuador de la vegetación comienza cuando el río desborda sobre el valle, aumenta hasta que la lámina de agua de inundación supera el sotobosque y la vegetación herbácea de los bañados, disminuyendo cuando las crecidas cubren totalmente este tipo de vegetación. Esta función atenuadora no es estática, dependiendo del desarrollo de la vegetación (cobertura, continuidad, densidad, altura, etc.) previo a las crecidas, lo que depende en gran medida de la duración de las crecientes.

Más del 95\% de la biomasa total de la biota del sistema fluvial está concentrada en la vegetación, por lo que cualquier modificación en su distribución y abundancia ha de tener efectos en la geomorfología en la oferta de hábitat y en las mallas tróficas de los ecosistemas fluviales. Nosotros entendemos que el análisis de la dinámica fluvial no debería restringirse al análisis del medio físico o del medio biótico, ni entender el funcionamiento del río únicamente por las diferencias de caudal. La comprensión de las interacciones entre los subsistemas y procesos del paisaje, permiten una perspectiva holística más útil para intentar modelos de funcionamiento y d e l manejo de los ríos.

\section{Referências Bibliográficas}

Basterra, I. (1999). Patrones naturales de 
variabilidad espacio-temporal del paisaje fluvial de una sección del Bajo Paraná, como base para la gestión de su manejo hídrico. Tesis de Maestría en Gestión Ambiental y Ecología. 94 p.

Bergman, D.L y Sullivan, C.W. (1963). Channel changes on Sandstone Creek near Cheyenne, Oklahoma. US. Geological Survey, Professional Paper 475c: 145-148.

Bravard, J.P. y Gilvear, D.J. (1996). Hydrological and geomorphological structure of hydrosystems. In: Petts G.E. Y Amoros, C. (eds.). Fluvial Hydrosystems. Chapman y Hall, London. Capítulo 5. 98-116.

Bruniard, E.D. (1996). Geografía de los climas y de las formaciones vegetales. Aporte para un modelo fitoclimático mundial. Las zonas térmicas y la vegetación natural. Editorial Universitaria de la Universidad Nacional del Nordeste. Corrientes, 330p.

(1999a). Los regímenes hídricos de las formaciones vegetales. Aportes para un modelo fitoclimático mundial. Editorial Universitaria de la Universidad Nacional del Nordeste. Corrientes, 382p.

(1999b). Clima. Caracterización climática del Nordeste de la Provincia de Corrientes (Propiedades: Puerto Valle, Garruchos y El Ciríaco). En: SHELL C.A.P.S. A. Forestal. Medio Ambiente. Evaluación del Impacto Ambiental de los Predios Forestales de 268p.

Casco, S.L. (2003). Poblaciones vegetales centrales y su variabilidad espacio-temporal en una sección del Bajo Paraná influenciada por el régimen de pulsos. Tesis Doctoral. Universidad Nacional del Nordeste. 1-189 y anexos.

Dolan, R., Howard, A. y Gallenson, A. (1974). Man's impact on the Colorado River in the Grand Canyon. Amer. Scientist. USA, 62:392-401.

FEDERAL ENVIRONMENTAL ASSESSMENT REVIEW OFFICE (FEARO). (1978). Ecological land survey guidelines for environmental impact analysis. Ecological Land Classification Series. Federal Environmental Assessment and Review Process. 13: 42p. Lands Directorate Environment Canadá.

Franceschi, E.A. y Lewis, J.P. (1979). Notas sobre la vegetación del valle santafesino del río Paraná (R.A.). Ecosur. Buenos Aires, 6,5582.

García, N.O. y Vargas, M.W. (1998). The temporal climatic variability in the "Río de la Plata" Basin displayed by the river discharges. Climatic Change. Dordrecht, 38:359-379.

Gradzī̄ski, R.; Baryla, M.; Doktor, M.; Gmur, D.; Gradzī̄ski, M.; Kedzior, A.; Paszkowski, M.; Soja, R.; Zielin̄ski, T. y Zurek, S. (2003).
Vegetation-controlled modern anastomosing system of the upper Narew River (NE Poland) and its sediments. Sedimentary Geology. Amsterdam, 157:253-276.

Graf, W.L. (1979). The development of montane arroyos and gullies. Earth Surface Processes. USA, 4:1-14.

Hearne, J.W. y Armitage, P.D. (1993). Implications of the annual macrophyte growth cycle on habitat in rivers. Regulated rivers. Inglaterra, 8:313-322.

Howard, A.D. y Dolan, R. (1981). Geomorphology of the Colorado River in the Grand Canyon. Journ. of Geology. Chicago, 89:269-298.

Junk, W.J. y Piedade, M.T.F. (1993). Herbaceous plants of the Amazon floodplain near Manaus: Species diversity and adaptations to the flood pulse. Amazoniana. Manaus, 12(3/4):467-484.

Kellerhals, R y Gill, D. (1973). Observation and potential downstrean effects of large storage projects in Northern Canadá. In Transaction of the eleventh Internat. Congr. of Large Dams, Madrid, España, vol.1.731-754.

King, N.J. (1961). An example of channel aggradation induced by flood control. US. Geological Survey Professional Paper 424B: 29-32.

Klimas, C.V., Martin C.O. y Teafor, J.W. (1981). Impacts of the flooding regime modification on wildlife habitats of bottomland hardwood forests in the lower Mississippi Valley. U.S. Army Eng. Waterways Exp. Sta. Tech. Rep., Vicksburg. 140p.

Lewis, J.P. y Franceschi, E.A. (1979). Notas sobre la dinámica de la vegetación del valle del río Paraná. Ecosur. Buenos Aires, 6:145163.

Moschini, V., Pompêo M.L. y Soares, J.J. (1995). Fenología de Scirpus cubensis na lagõa do Infernão (Brasil). Acta Limnol. Brasil. Río de Janeiro, 7:1-9.

Neiff, J.J. (1978). Fluctuaciones de la vegetación acuática en lagunas del valle del río Paraná en la transección Paraná-Santa Fe, entre 1970 y 1977. Physis. Buenos Aires, 38(95):41-53.

(1986). Las grandes unidades de vegetación y ambiente insular del río Paraná en el tramo Candelaria-Itá Ibaté. Rev. Cienc. Nat. Litoral. Santa Fe, 17(1):730.

(1990). Ideas para la interpretación ecológica del Paraná. Interciencia. Venezuela, 15(6):424-441. 
(1999). El régimen de pulsos en ríos y grandes humedales de Sudamérica. In Malvárez, A.I. (ed.). Tópicos sobre humedales subtropicales y templados de Sudamérica. Universidad de Buenos Aires. 97-146.

(2001). Diversity in some tropical wetland systems of South America. In Gopal, W.; Junk, J. Y Davis, J.A. (eds.) Biodiversity in wetlands: assessment, function and conservation. Backhuys Publishers, Leiden, The Netherlands, 157-186.

(2003). Planicies de inundação são ecótonos? In HENRY, R. (ed.). Ecotonos nas interfaces dos ecosistemas aquáticos, Capítulo 2. RIMA, San Carlos, Brasil. 32-47.

Neiff, J.J. y Neiff, M. (2003). Pulso, software para análisis de fenómenos recurrentes. Dir. Nac. de Derecho de Autor No 236164 (Argentina) Buenos Aires, 17 de febrero. http://www.neiff.com.ar.

Neiff, J.J. y Poi Neiff, A. (2002). Connectivity processes as a basis for management of aquatic plants. En: Magela Thomaz, S. Y Bini L.M. Ecologia e Manejo de Macrófitas Aquáticas (eds.). Universidade Estadual de Maringá, Maringá, Brasil, 40-58.

Neiff, J.J., Iriondo, M.H. y Carignan, R. (1994). Large Tropical South American Wetlands: An Overview. Proc. of the Internat. Workshop on the Ecology and Management of Aquatic-Terrestrial Ecotones. 156-165.

Newson, M.D. (1980). The erosion of drainage ditches and its effect on bed-load yelds in mid-Wales: reconnaissance case studies. Earth Surface Processes. USA, 5(3):275290.

Northrop, W.L. (1965). Republican River channel deterioration. U.S. Dept. of Agriculture, Miscellaneus Publ. 970: 409-424.

Orfeo, O. (1995). Sedimentología del río Paraná en el área de confluencia con el río Paraguay. Tesis Doct. Univ. Nac. de La Plata. 286p.

(1996). Geomorfología del sistema fluvial Paraguay-Paraná en el área de su confluencia. Actas del XII Congreso Geológico Argentino y III Congreso de Exploración de Hidrocarburos: 131-147p.

Orfeo, O. y Stevaux, J. (2002). Hydraulic and morphologic characteristics of middle and upper reaches of the Paraná River (Argentina and Brazil). Geomorphology. Liverpool, 44(3/4):309-322.

Petrere, M. y de Brito Ribeiro, M.L. (1994). Impact of a large tropical Hydroelectric dam. The case of Tucuruí in the middle River Tocantins. Acta Limnol.Brasil. Río de Janeiro, 5:123-134.

Petts, G.E. (1982). Channel changes within regulated rivers. In Adlam, b.H., Fenn, C.R.
Y Morris, L. (eds) Papers in Earth Studies. Geobooks, Norwich, (U.K.), 117-142.

Poi de Neiff, A., Neiff, J.J.; Orfeo, O. y Carignan, R. (1994). Quantitative importance of particulate matter retention by the roots of Eichhornia crassipes in the Paraná floodplain. Aquatic Botany. Amsterdam, 47:213-223.

Schnack, J.A.; De Francesco, F.; Galliari, F; Neiff, J.J.; Oldani, N.; Schnack, E. y Spinelli, G. (1995). Estudios ambientales regionales para el proyecto de control de inundaciones. Informe final. Minist. del Interior (SUPCE), Buenos Aires. Ecological Processes and acumulative impacts. J. G. Gosselink, Lee, L. C. y Muir, T. A. Lewis Publ. Chelsea; MI. 1388.

Tattar, T.A. (1972). Effects of inundation on trees. USDA For. Serv., N.E. Area State and Priv. For. P-72-4: 6p.

Taylor-Golder-Consular-Connal. (1996). Evaluación del impacto ambiental del mejoramiento de la hidrovía ParaguayParaná. Módulo B2. Diagnóstico Integrado preliminar. Volumen 5.

Teskey, R.O. y Hinckley, T.M. (1977). Impact of water level changes on woody riparian and wetland communities. U.S. Fish and Wildlife Service Off. of Biol. Serv. 77/58, 77/59; 77/60; 78/87. 78/88; 78/89.

Turner, R.M. y Karpiscak, M.M. (1980). Recent vegetation changes along the Colorado River between Glen Canyon Dam and Lake Mead, Arizona. U.S. Geological Survey Professional Paper 1132: 125p.

-Taylor-Golder-Consular-Connal. (1996). Evaluación del impacto ambiental del mejoramiento de la hidrovía ParaguayParaná. Módulo B2. Diagnóstico Integrado preliminar. Volumen 5.

Wende, R. y Nanson, G. (1998). Anabraching rivers: ridge-form alluvial channels in tropical northern Australia. Geomorphology. Liverpool, 22: 205224.

Whitlow, T.H. y Harris, R. (1979). Flood tolerance in plantas: a stere-of-the-art review. U.S. Army Eng. Waterways Exp. Stn. TR E-79-2. Vicksburg, MS. 16lp.

Whittaker, R.H. (1978). Ordination of Plant Communities. W. Junk bv Publishers The Hague, Boston. 388p

Wolman, M.G. y Gerson, R. (1978). Relative scales of time and effectiveness in watershed geomorphology. Earth Surfac Processes. USA, 3:189-208. 